\title{
Are rodent models of Parkinson's disease behaving as they should?
}

\author{
Siv Vingill ${ }^{1,2}$, Natalie Connor-Robson ${ }^{1}$, Richard Wade-Martins ${ }^{1}$ \\ ${ }^{1}$ Oxford Parkinson's Disease Centre, University of Oxford, South Parks Road, Oxford OX1 3QX, UK \\ ${ }^{2}$ Corresponding author
}

\begin{abstract}
In recent years our understanding of Parkinson's disease has expanded both in terms of pathological hallmarks as well as relevant genetic influences. In parallel with the aetiological discoveries a multitude of PD animal models have been established. The vast majority of these are rodent models based on environmental, genetic and mechanistic insight. A major challenge in many of these models is their ability to only recapitulate some of the complex disease features seen in humans. Although symptom alleviation and clinical signs are of utmost importance in therapeutic research many of these models lack comprehensive behavioural testing. While non-motor symptoms become increasingly important as early diagnostic markers in PD, they are poorly characterized in rodents. In this review we look at well-established and more recent animal models of PD in terms of behavioural characterization and discuss how they can best contribute to progression in Parkinson's research.
\end{abstract}

\section{Introduction}

200 hundred years have passed since James Parkinson first described Parkinson's Disease (PD); a progressive neurodegenerative disorder that affects approximately $2-3 \%$ of people aged over 65 [1, 2]. The disease is normally diagnosed on the clinical presentation of bradykinesia as well as one of the other cardinal symptoms; resting tremor, rigidity and postural instability. Although PD is primarily considered to be a motor disorder, it is evident that there are numerous non-motor symptoms such as hyposmia, constipation, sleep disorders and depression [3]. The main symptoms of PD arise due to the loss of dopaminergic neurons in the substantia nigra pars compacta (SNpc), which causes imbalance of the nigrostriatal pathway. However, pathology across the rest of the brain is thought to be responsible for some of the non-motor symptoms. Importantly, Lewy bodies, one of the hallmarks of PD, are highly enriched in the brains of affected individuals [4]. These are proteinaceous inclusions that are primarily formed of aggregated forms of $\alpha$-synuclein [5], but also contain a variety of different proteins and membranous components [6].

Many different genes have been associated with PD, either through pedigree genotyping, linkage or genome-wide association studies [7]. Mutations in these genes cause familiar variants of PD or are, along with others, considered risk factors in sporadic disease. The majority of Parkinson's cases, however, remain idiopathic. This shows a combination of genetic and environmental contribution to PD pathology, which along with the diverse array of symptoms and key pathological hallmarks, makes PD a difficult disease to fully recapitulate in animal models. An ideal PD model is thought to reflect a progressive and age-dependent disease presentation with key motor and non-motor symptoms. Pathologically the model should include loss of dopaminergic neurons in the SNpc and reduced dopamine content in the striatum as well as neuroinflammation and aggregation pathology reminiscent of Lewy bodies. These criteria are rarely fully met, yet a plethora of different PD models exists, ranging from Drosophila to non-human primates. Rodents are however, often preferred due to their relative rapid breeding cycle and mammalian genome.

The rodent models of PD can be broadly divided into environmental models, including the widely used MPTP mouse and 6-OHDA rat models, and genetic models with knock-in and knock-out rodents based on known PD-associated genes. The induced aggregation models represent a middle ground 
and centres on the progressive accumulation of $\alpha$-synuclein in PD brains. Most of these models have been assessed in terms of motor impairment and neuropathology, with clear variability between studies.

\section{Environmental models of Parkinson's disease}

The now well-characterised MPTP model was identified when a batch of contaminated heroin resulted in the hospitalisation of users with irreversible PD [8]. MPTP is converted via astrocytes to MPP+, which targets the mitochondrial complex I specifically in dopaminergic neurons through selective dopamine transporter (DAT) import and thus results in cell death [9]. In some mice strains MPTP treatment results in acute non-progressive dopaminergic cell loss accompanied by reductions in striatal dopamine, while rats and other strains are more resistant to its effects [10]. Motor tests have been widely employed, but the severity depends on the treatment regime, with many studies reporting no or transient motor phenotypes. Additionally, transient effects on sleep [11, 12] and colon motility [13] as well as cognitive disturbances $[14,15]$ have been found in the acute MPTP model. The main caveat of the MPTP models has been the acute dosing, which gives sickness behaviour and celldeath within hours [10]. In contrast, chronic exposure to MPTP induces a more progressive neuronal loss and some studies report inclusion pathology in remaining neurons [16-19], although this is controversial [20-22]. Many of these studies also report progressive motor dysfunction [17, 18, 20, 23, 24] with Schintu et al. observing additional olfactory deficits [25]. The chronic and sub-acute MPTP models could hence be more suitable for treatments as it gives a larger window of early symptomatic stages in which to apply a drug.

The use of 6-hydroxydopamine (6-OHDA) provides a more readily reproducible lesion toxin model with high levels of SNpc dopaminergic cell death within the first few days following injection. Additionally, the level and acuteness of cell death can be modulated depending on the injection site within the nigro-striatal system [26]. The unilateral 6-OHDA injection produces a well-described stereotyped rotational behaviour upon dopamine agonist treatment, which has provided the basis for many neuroprotective studies [27]. In addition to showing rotarod and paw reaching motor impairments this model has also demonstrated more complex behavioural and cognitive impairments including extended operant box [28, 29] and Morris water maze testing [30]. An additional advantage of the 6-OHDA rat model is its ability to portray dyskinesias, a common side-effect of L-dopa treatment [27]. The emergence of abnormal involuntary movements after treatment with dopamine agonists can aid in preventive and symptomatic drug evaluation [31]. In summary, the 6-OHDA model provides selective dopaminergic cell death with highly replicable lesions, but is limited in terms of progression and modelling the age-related effects of PD and does not show inclusion pathology.

The rotenone rat model is based on a pesticide that also inhibits Complex I [32]. This model has the advantage of being able to induce loss of dopaminergic neurons upon systemic exposure, with $\alpha-$ synuclein positive inclusions present in nigral neurons [33], but shows high individual variability depending on administration protocol [34]. These drawbacks have lately been addressed with lower doses and new administration routes. Many motor tests have been employed in this model showing deficiencies in endurance, posture and activity, for further details see Johnson et al [34]. Systemic toxicity has been an issue [35], but in the newer models the motor symptoms have correlated with dopaminergic neuron loss and could be reversed by apomorphine, a dopamine agonist [36]. Loss of smell and constipation are two of the earliest symptoms of PD and the mucous membranes of the nose and gut have been suggested as nucleation sites for environmentally introduced pathology. Notably, intragastric infusion of rotenone led to widespread synuclein pathology with inclusions seen in the enteric nervous system as well as in the brainstem and substantia nigra [37]. The systemic administration of rotenone hence more accurately portrays environmental exposure than toxins injected directly into the brain such as 6-OHDA. Although this model is largely used for its motordisturbances, some studies have reported non-motor symptoms including sleep disturbances, gastric impairment, depressive/anxiety-like behaviour and reduced sense of smell [34]. 
Neuroinflammation is an important feature found in sporadic PD [38-40], and both the MPTP [41, 42] and the rotenone [43] model feature this element to some degree through invasion of T-cells, microglial activation, astrocytosis and cytokine response, adding to the face validity of these models [44]. In addition, the treatment of rodents with lipopolysaccharide (LPS), a potent microglial activator, induces toxicity to dopaminergic neurons in vivo both in mouse and rat models [45]. The LPS model notably features amphetamine induced rotational behaviour [46] as well as decreased movement parameters in automated tracking analysis [47]. These changes were transient in young rats, but failed to improve in older rats. In addition the LPS model has been used to accentuate phenotypes in other models, both genetic and environmental, which will be discussed later in this review.

Other toxin-induced models are able to induce loss of dopamine, but are less comprehensive than the aforementioned models [26]. Collectively, the toxin models have been fundamental to the development of the existing symptomatic treatments of PD [27], but show difficulties in predicting efficacy of disease-modifying treatments due to their acute nature [48]. However, the advances in treatment regimes and delivery routes have sparked new interest in the toxin models as progressive models. In addition, these models can be combined with genetic models to investigate geneenvironment interactions in PD.

\section{Genetic models of Parkinson's disease}

Due to the multifaceted nature of PD, many genes have been associated with the disease, but with stringent criteria applied, only six genes have been confirmed as monogenic PD variants [49]. These comprise of the recessively inherited Parkin, PINK1 and DJ-1, as well as the autosomal dominant SNCA, LRRK2 and VPS35.

\section{Parkin, PINK1 and DJ1}

Parkin, PINK1 and DJ-1 are all thought to act in parallel in the regulation of mitochondrial quality in the cell [50]. Due to their recessive inheritance pattern, knock-out mice have been favoured to model loss-of-function of the proteins. Although some studies have reported mild motor impairment in individual DJ-1, Parkin and PINK1 knockout mice, no neurodegeneration has been found [51-54]. Notably, a triple knockout of PINK1, Parkin and DJ-1 show no loss of SNpc neurons accompanied by increased striatal dopamine in aged mice [55]. Rat models might show more promise as a recent study thoroughly characterized individual DJ-1, PINK1 and Parkin knockout rats and found that DJ-1/and PINK 1 - rats showed progressive motor defects accompanied by a $50 \%$ loss of SNpc neurons at 8 months of age [56]. Parkin ${ }^{-/-}$rats, in contrast, were indistinguishable from wild-type controls. Despite the relatively minor behavioural disturbances and the lack of cell loss, these models have provided mechanistic insight into pathways disturbed in PD. In addition, both behavioural and pathological findings suggest that these models might represent an early disease stage. This can be used both to investigate pre-motor symptoms and genetic susceptibility either alone or in combination with environmental models.

\section{LRRK2}

One of the most common forms of genetic PD is caused by mutations in LRRK2 and is symptomatically indistinguishable from the sporadic disease [2]. LRRK2 encodes a multidomain protein with both kinase and GTPase functions, whose mutations are thought to influence its kinase activity as well as its association with interaction partners $[57,58]$. Most models therefore focus on expressing mutated versions of the protein. Some traditional knock-in models expressing either the G2019S [59] or R1441C [60 ] mutations have shown progressive reduction in dopamine release and altered dopaminergic neurotransmission respectively, but little has been shown in these models in terms of behaviour or cognitive deficits. Recent models take advantage of bacterial artificial chromosomes (BAC), which allows for large genomic segments to be effectively inserted into the genome. Thus, the endogenous promotor as well as upstream and downstream regulatory elements 
can be included in the model, which ensures better temporal and spatial expression. These models have revealed relatively low expression of LRRK2 in SNpc dopaminergic neurons [61, 62], yet agreement has been reached that overexpression of G2019S [62,63] or R1441G/C LRRK2 leads to a reduction in dopamine release accompanied by impaired motor function [61, 64], which can be corrected by administration of L-Dopa [61]. Overexpression of wild-type LRRK2 however, yields inconsistent results both in regards to striatal dopamine release and behavioural activity level in these mice [61-63, 65]. Interestingly, none of these models report any dopaminergic cell loss or a-synculein pathology, but some report tau pathology, which is known to occur in the brains of LRRK2 patients [62, 64]. The discrepancies between these studies may arise from differences in LRRK2 species, human vs. mouse, or expression levels of the transgene. Little has been shown in these models in terms of non-motor dysfunction apart from age related decline in spontaneous alternation reported in G2019S and R1441C rats [61]. Knockout models of LRRK2 have also been studied in the hope of revealing its normal function, but have been unfruitful $[66,67]$. No alterations in the nigrostriatal system have been demonstrated, even in aged rodents, which may be due to the remaining expression of LRRK1 or other compensatory methods. A question still remains whether LRRK2 mutations induce a purely gain of function phenotype, hence broader behavioural characterization of both knock-in and knock-out models is required.

\section{VPS35}

VPS35 encodes vacuole protein sorting-35, a protein essential for the retrieval of membrane proteins from the Golgi apparatus to the endoplasmic reticulum [68]. VPS35 knockout mice are embryonically lethal [69], therefore heterozygous and conditional knockout mice using the DAT-promotor have been established [70,71]. These mice are viable and show loss of neurons in SNpc preceded by a decrease in striatal dopamine levels. They also show motor deficits, which emerge earlier and are more severe in the conditional knockout than in the heterozygous mice. In addition, age-dependent $\alpha$ synuclein accumulation could be seen in the midbrain of both mouse-lines. Further studies of this model are warranted, but it represents an interesting genetic model with a comprehensive phenotype.

\section{Additional genetic models}

Heterozygous mutations in GBA, which encodes the lysosomal enzyme glucocerebrosidase (GCase), are the most common genetic risk factor for developing PD [72]. In cellular neuronal models of PD heterozygous point mutations in GCase lead to aberrant protein processing, ER stress, perturbed autophagy and lysosomal dysfunction, which impact on normal $\alpha$-synuclein degradation [73]. In synuclein mouse models, increase of GCase levels or stability can alleviate some of their phenotypes, including an improvement in motor testing and a reduction in a-synuclein pathological load [74, 75]. Conversely, introducing the GBA heterozygous L444P mutation in mice overexpressing human A53T a-synuclein exacerbates motor impairment on the rotarod [76]. Currently none of the GBA PD models has undergone extensive behavioural or biochemical phenotyping, which is required to gain in depth knowledge of how GCase influences the development of PD.

Apart from the well-established PD genes, atypical cases of familial Parkinsonism, such as ATP13A2 $[77,78]$ and FBXO7 [79], have recently been modelled in rodents resulting in strong behavioural phenotypes accompanied by neuropathological changes. Collectively these models might provide useful mechanistic insight into subsets of Parkinsonian disorders, which can be transferred to the broader sporadic cases. In addition specialized treatments targeting these familiar variations will be highly beneficial for the patients affected.

An interesting genetic model is the MitoPark mouse [80], which is based on findings from toxic and genetic models implicating mitochondrial dysfunction as a major pathway disturbed in PD. These mice have the mitochondrial transcription factor A (Tfam) knocked out under the DAT promoter, giving dopaminergic neurons in these mice chronic mitochondrial dysfunction that leads to progressive loss of SNpc neurons. Despite this, the mice develop normally until 6 weeks of age when they show 
emerging motor impairment that progresses to paralysis over the course of 30 weeks [81]. Interestingly, the motor symptoms have been shown to be preceded by mild cognitive impairment [82]. This model thus recapitulates some of the progressive nature of PD, including early symptoms of the disease, but is limited to specific neurons and fails to capture the widespread aggregation pathology.

\section{$\alpha$-synuclein}

Although $\alpha$-synuclein is responsible for the main histopathological hallmarks of PD and it is clear that mutations, duplication or triplication of the gene result in early onset genetic forms of PD, its function as yet, is poorly defined [83].

\section{Knock-in and knock-out models}

Despite being a highly abundant protein, constitutive knockout of the gene only causes relatively minor phenotypes with two different a-synuclein knockout mouse models showing alterations in synaptic dynamics but no behavioural phenotypes [84, 85]. The synuclein family includes two additional homologues; $\beta$ - and $\gamma$-synuclein, which may compensate for the loss of $\alpha$-synuclein. In the absence of any synucleins mice demonstrate a hyperactive phenotype despite having significant reductions in striatal dopamine [86]. A recent study in which all combinations of synuclein knockout mice were compared, importantly on the same genetic background and housing conditions, it was reported that normal striatal dopamine content was dependent on the presence of $\alpha$-synuclein whereas $\beta$-synuclein seems to be important in maintaining normal motor function during aging [87]. Due to the apparent lack of phenotypes in these knockout mice many have concluded that $\alpha$ synuclein does not have a substantial role in dopaminergic survival and that its role can be compensated. However, these studies seem consistent with a role for $\alpha$-synuclein in the normal release of synaptic vesicles.

A number of overexpression models of $\alpha$-synuclein with expression driven by various promotors including PDGF $\beta$, PrP and Thy-1 have been produced [88-91]. Although all of these models demonstrate behavioural and neuropathological phenotypes, the Thy- 1 model has been most extensively characterised and recapitulates various features of PD, including astroglial and microglial activation as well as increased levels of TNF- $\alpha$, TLR1,4 and 8 [92]. Importantly, this model demonstrates phosphorylated and proteinase $\mathrm{K}$ resistant aggregates of $\alpha$-synuclein throughout the brain and further pathology in the enteric nervous system [91]. Extensive behavioural characterisation has also been carried out in this model revealing deficits in both fine and gross motor behaviours. In addition, early non-motor phenotypes are prevalent with the mice showing impaired olfactory function, disrupted circadian rhythms, colonic deficits and cognitive disturbances [91]. The aforementioned promoters drive high expression throughout the central nervous system including motor neurons, which complicates interpretation of motor phenotypes. To specifically drive expression in dopaminergic neurons, synuclein has been expressed under the tyrosine hydroxylase promoter [93, 94]. However, no strong phenotypes were observed; most likely due to the weak nature of this promoter.

Models expressing mutant $\alpha$-synuclein or truncated variants, as found in Lewy bodies, have also been developed [89, 90, 95-98]. On a whole, these models present with similar phenotypes to those described above with alterations in striatal dopamine, motor dysfunction, neuroinflammation and pathological inclusions more widely reported than in wild-type overexpression models.

\section{Genomic DNA locus (BAC/PAC) transgenics}

A different genetic approach is the BAC transgenics where spatially and temporally relevant $\alpha$ synuclein gene expression is achieved by the endogenous promoter. These animals show motor phenotypes as well as gastrointestinal, olfactory and sleep disturbances [99]. However, expression of 
human $\alpha$-synuclein on the background of endogenous mouse $\alpha$-synuclein could confound the interpretation of these findings. Janezic et al produced a BAC transgenic model harbouring the entire human $\alpha$-synuclein locus expressed in endogenous $\alpha$-synuclein knockout mice [100]. These mice show an early and persistent reduction in the release of dorsal striatal dopamine followed by progressive motor impairment. Constipation, a key non-motor symptom, was also evident in these mice from a young age and at 18 months of age they show a $30 \%$ reduction in dopaminergic neurons of the SNpc. In addition the BAC technology has been used to express mutant versions of $\alpha$ synuclein, E46K [101] and A30P [102], in rats and mice respectively, with the animals showing rather mild phenotypes. The similar PAC genomic locus vector was used to drive the overexpression of A53T and A30P $\alpha$-synuclein in mice, causing early enteric dysfunction and motoric disturbance in the case of the A53T mutant [103].

These models have demonstrated that changes in the normal expression of $\alpha$-synuclein are enough to drive behavioural, non-motor and neurotransmission phenotypes. Despite this, it fails to induce the hallmark aggregation pathology seen in human patients.

\section{Viral overexpression models}

Given a-synucleins prominent role in Lewy bodies, the genetic models have been complemented by viral vectors used to overexpress wild-type or mutant $\alpha$-synuclein directly in regions of interest [104]. Most studies have focused on rAAV mediated overexpression in Sprague-Dawley (SD) rats where protein expression is increased by approximately $2-5 x$ the amount of endogenous rat $a$-synuclein [105-108]. The pathology in these models shows aggregation of $\alpha$-synuclein in the SNpc as well as other brain regions. These differ from the classical Lewy bodies, with only a few studies showing filamentous and/or phosphorylated aggregates [106, 109, 110]. Most studies, however, report degeneration of neurons in the SNpc ranging from 20-80\% as reviewed in Volpicelli-Daley et al [111], with the variability largely depending on vector, serotype and titer of the virus [108, 112]. Several of the viral overexpression models have assessed the impact of PD relevant mutations in $\alpha$-synuclein, but the results have been varied. One study has found the A53T mutant to give little or no nigral toxicity [113]. However, other studies have shown the A53T mutation to give a stronger [114], or similar phenotype to wild-type a-synuclein overexpression, including behavioural deficits that are correctable using L-dopa and ubiquitin positive $\alpha$-synuclein aggregates $[115,116]$.

These models are all based on a unilateral injection with pathology mostly seen in the ipsilateral striatum. The dopamine agonist apomorphine can be used to primarily increase activity in the unaffected side, which leads to rotational behaviour. This induced phenotype poorly represents PD symptoms, however, depending on neuronal loss, most studies report an increase in such rotations [111]. Additional tests have revealed gait alterations [117] and forepaw asymmetry in rAAV injected rats [112, 118, 119], which has been rescued upon L-Dopa administration [120]. In terms of nonmotor symptoms, one group found deficiencies in ultrasonic vocalizations [112], while a recent study showed an increase in avoidance behaviour connected to impairment of cholinergic striatal neurons [121]. In addition to the rat models some mice models have also used viral vector injection. Most of these studies find a loss of dopaminergic neurons reminiscent of the rat studies [115, 122-124]. Two studies assessed behaviour and found increased forelimb asymmetry, and additional motor impairment across several tests [115, 124]. Similar to the other unilateral injection models, these models offer the advantage of using the contralateral hemisphere as an internal control, but subtle behavioural features can be overlooked due to compensation from the unaffected hemisphere.

\section{$\alpha$-synuclein protein spread models}

In recent years evidence has accumulated supporting the hypothesis that $\alpha$-synuclein pathology can spread in vivo in a "prion-like" fashion. Braak and colleagues observed that the Lewy body pathology is found in different brain regions corresponding to disease progression [4]. In addition it was shown that transplants of neuronal grafts to PD patients had accumulated aggregations 20 years after 
transplantation [125]. Experiments in cell-culture have further confirmed that $\alpha$-synuclein can be secreted from and taken up into neurons [126] and new rodent models are currently evolving to investigate the spread of $\alpha$-synuclein in vivo. In this respect it has been shown that brain tissue from symptomatic mice or synucleinopathy patients can aggravate pathology when injected into asymptomatic M83 transgenic A53T mice [127-129] or induce pathology in wild-type mice [130, 131]. However, the use of biological material makes it unclear if $\alpha$-synuclein is the acting agent and therefore pre-aggregated recombinant $\alpha$-synuclein, so called pre-formed fibrils, are also used [132143]. The initial study reported cell-loss in the SNpc of $35 \%$ in non-transgenic mice [132], but this has been inconsistent across laboratories [143]. When aggregates are reported in $\alpha$-synuclein spread models they stain positive for phosphorylated $\alpha$-synuclein, p62 and ubiquitin to various degrees, hence resembling the composition of Lewy bodies. Further contributing to relevant PD pathology; astrogliosis and microgliosis are reported in some [127, 136], but not all [131], of these models. Different administration routes have shown that $\alpha$-synuclein fibrils can spread from the periphery [144, 145] and gastrointestinal exposure to brain homogenates of PD patients or recombinant forms of $\alpha$ synuclein have shown transport via the vagal nerve to the brain in rats [146]. Some studies show motor impairment post-injection [132, 134, 138] and a recent study with $\alpha$-synuclein oligomers infused into the cerebroventricular space of mice in addition showed the presence of both olfactory and anxiety symptoms [147]. Direct exposure of fibrillar mouse and human $\alpha$-synuclein to the olfactory bulb has shown widespread pathology $[142,148]$ accompanied by a decline in olfactory retention, but without motor or anxiety deficits [149]. In rats motor deficits [144] as well as a decrease in ultrasonic call frequency, duration and vocalisation amount has been shown [150]. Follow up studies stress that considerations need to be taken into account for these models regarding the material injected. Different results have been obtained with mouse vs. human and oligomeric vs ribbon and fibrillar $\alpha-$ synuclein as well as with varying concentrations and fibrillar length [138, 144, 149, 151]. In addition the strain and gender of mice might influence their susceptibility to $\alpha$-synuclein pathology [111, 134]. Taken together, these models show promise in respect to therapeutic development focused on preventing a-synuclein pathology.

\section{Future directions of PD rodent modelling}

Recent years have seen a large increase in animal models for Parkinson's disease with rodent models dominating the field. Each model has its own strengths and limitations that are important to be aware of when designing a study. Administration of environmental agents leads to acute cell-death with limited aggregation pathology and can therefore be said to represent an advanced disease state. These models have been widely used to study symptomatic or neuroprotective treatments, but present only a short time window for manipulation of disease-progression. However, as improved dosing schemes and routes of administration are implemented, this window might expand.

Genetic knock-in/knock-out models seek to replicate familiar variants of PD under the assumption that these share similar disease mechanisms to idiopathic PD. These models have given valuable insight into pathways affected in PD and pointed to specific drug targets, but gross motor symptoms have been absent in many models. Yet expanded testing show subtle phenotypes present in these mice suggesting we might be modelling an early phenotype. It is estimated that motor symptoms in patients only occur after a loss of $30-50 \%$ of striatal dopamine [152-154]. The so called die-back mechanism suggests that synapses and axons degenerate before the cell-bodies in the SNpc are affected, which might present a therapeutic window given that reliable diagnostic criteria are available. Many of the genetic models show disturbances in synaptic machinery [155], which are thought to precede neuronal loss in PD. Indeed, some models show early elevations in striatal dopamine content, which could result in toxicity at the synapse $[56,156]$. This abundance of dopamine results in hyperactive behavioural phenotypes [156] mirroring the elevated dopamine levels seen in LRRK2 carriers prior to the onset of symptoms [157]. The notion that incorrect storage of dopamine leads to cell toxicity is further supported by increased PD risk in people carrying variants of the dopamine transporter gene [158] and progressive cell death in mouse models with reduced vesicular monoamine transporter 
(VMAT) expression [159]. The lack of severe phenotypes in many genetic models might in this way provide a more accurate representation of the early disease state than the environmental models.

Previous genetic models are predominantly made in mice due to the relatively easy manipulation of their genome and embryonic stem cells. However, with the newly developed techniques in gene editing, the hurdle of making genetic rat models is also largely overcome [160]. The move to rat models gives more severe phenotypes for the PINK-/- and DJ-1 knockouts [56] as well as the SNCA BAC model [161]. Due to their larger size, imaging such as PET and fMRI is more feasible in such models. In addition they allow access to a more extensive range of behavioural tests due to their ability to perform more challenging cognitive tasks [160].

Induction of $\alpha$-synuclein pathology by injection of aggregated protein is enough to cause behavioural deficits with several studies also showing cell death and decline in striatal dopamine. Although caution should be taken when interpreting the pathological findings in these models [162], they contribute to the hypothesis that synucleinopathies can be viewed as "prion-like" disorders [163]. Like some of the toxin models, they offer the ability to investigate the olfactory and gastrointestinal systems as initiation sites for the disease, but it is clear that strict biochemical accuracy is required in the production of $\alpha$ synuclein that is to be injected. In addition to traditional motor features of PD, these models also focus on early detection of symptomatic behaviour, such as olfaction, paralleling progression of pathology.

One challenge present across models is the apparent higher resistance of rodent dopaminergic neurons to degeneration compared to humans. For instance, mouse $\alpha$-synuclein naturally harbours the A53T mutation, which causes PD in humans. Rodents also seem more resistant to toxins, with rats and certain mouse strains not responding significantly to MPTP. The lack of penetrance for riskfactor genes and the fact that less than $10 \%$ of PD cases can be attributed to genetic causes suggest heavy environmental influence. A plausible paradigm in PD research is therefore the multiple hit concept [164], where different pathways contribute to cell-death. Several models now take advantage of the combination of genetic vulnerability and toxic insult to the cell. For instance it has been shown that DJ1\% mice show an increased vulnerability to MPTP compared to control mice $[165,166]$. Additionally PINK1 $\%$ mice show increased degeneration of dopaminergic neurons upon overexpression of $\alpha$-synuclein compared to control [167]. Interestingly, $\alpha$-synuclein may be able to modulate MPTP toxicity with reports of resistance to the toxin in $\alpha$-synuclein KO models $[18,168$ 170]. Vice-versa, the overexpression of $\alpha$-synuclein combined with rotenone exposure produced increased behavioural impairment and neuronal loss [171]. The recent induced a-synuclein aggregation model has also been combined with LRRK2 G2019S overexpression in rats, showing that these rats are more vulnerable to synuclein aggregation [172]. Also the induction of neuroinflammation has been shown to enhance PD features in other models. Pre-treatment of rodents with polyinosinic;polycytidylic acid [poly(l:C)], a viral agent, sensitizes DA neurons to subsequent 6OHDA [173] or paraquat [174] treatment, most likely through microglial activation. In terms of genetic models it has been shown that Parkin ${ }^{-1-}$ mice show an increased vulnerability to LPS [175]. The same is true in mice that express wild-type or A53T mutant $\alpha$-synuclein where LPS causes neuronal death $[176,177]$. In addition LRRK2 has been found to be necessary for microglial activation upon LPS treatment, hence underscoring the possible role of LRRK2 as an inflammatory modulator [178]. The multiple hit paradigm hence opens new avenues for established models with limited pathology and might trigger cell death even in the more robust rodent dopaminergic neurons.

The multitude of rodent PD models calls for strict practises when it comes to characterisation. It has been extensively shown that housing practises, experimental design and mixed background of mouse and rat lines can have great effect on animal behaviour $[179,180]$. In addition small variations in protocols and negative results are often under-reported. The lack of standardization is also a problem when designing the models; different promotors and expression systems define the genetic models while overexpression and toxin models are heavily influenced by dosing regimens, expression quantities and routes of delivery. The lack of reproducibility is a problem especially where phenotypes 
are subtle or individual variability is high. Many of the behavioural tests currently in use are also conducted differently across laboratories but appreciable efforts are made to standardise the reporting of all studies involving in vivo animal experiments with the ARRIVE guidelines adopted by many journals [181]. Traditional behavioural phenotyping is also heavily dependent on the experimenter and often exposes the rodent to stressful situations [182]. Advances in technology allow us to test rodents in more replicable and relaxed settings. Phenotyping cages are getting increasingly better and offer readouts of a wide array of simple and complex behaviours such as circadian rhythms, spontaneous activity, social contact and feeding behaviour [183]. In addition newer equipment offers more accurate readouts of the classic motor phenotypes. Automated gait analysis systems can detect subtle alterations in gait that might develop before identification is possible by cruder motor tests such as the rotarod and pole test $[184,185]$. In addition more advanced memory tests are being developed, mostly for fields such as Alzheimer's disease, which should clearly be extended to model the cognitive features seen in PD [186].

These paradigms can broaden the description of behaviours in rodent PD models; exemplified by a recent study that provided a more accurate time course for the motor impairment in A53T overexpressing mice, while revealing early deficiencies in grooming behaviour and spatial memory [187]. The non-motor symptoms in PD are often poorly characterized, but in many cases precede motor symptoms by decades and are reported by patients as more incapacitating in their daily life [188]. These symptoms include sleep disturbances, anosmia, constipation, depression, anxiety and other cognitive deficits. Taylor and colleagues have accurately suggested a paradigm of tests that includes motor as well as non-motor tests to give a more comprehensive picture of the disease progression in PD models [189]. To implement a similar extensive testing regime in both established and emerging rodent models is crucial due to the obvious burden non-motor symptoms cause PD patients.

The high failure rate of drugs in neurodegeneration [190], gives incentive for scientists to develop well characterized animal models to increase predictive value of preclinical trials. A meta-study comparing clinical outcome to preclinical assessment in animals found therapies tested in more than one model to have a higher success rate [48]. This study also highlights that most drug treatments are given to young male mice and rats, which is problematic as PD is an age-related disorder. Brain plasticity is reduced with age, which is not factored into many genetic models where defects are present throughout development. Therefore conditional models may represent a way to investigate changes in an aged nervous system, such as the tamoxifen inducible $\alpha$-synuclein knockout mouse [191]. In addition, a single timepoint is often used to determine outcome, whereas longitudinal studies would clearly be advantageous. For instance, the toxin models MPTP and 6-OHDA were heavily favoured in preclinical studies due to their relatively low cost and speed of degeneration. In many of these studies treatments aimed at disease-modification were given prior or simultaneous to the toxic event. This is a poor reflection of PD progression and disease-modifying therapies should be tested in progressive models at early symptomatic stages. Zeiss et al. conclude that the face-validity of therapeutic trials can be improved by cross-validation within a model in terms of age and gender as well as across different animal models [48]. Combined with extensive behavioural testing that more accurately represents the clinical situation, this could improve the prediction of therapeutic efficacy in humans.

\section{Conclusion}

Although no single model is currently able to recapitulate Parkinson's disease as seen in humans we can target different aspects of this complex neurodegenerative disorder by utilising multiple models. Mechanistic insight is often based on animal models and is valuable to identify targets for therapeutic intervention. However, for the life quality of patients, symptom alleviation and prevention of medication side-effects remains a highly sought after goal. It is therefore paramount that new treatments are evaluated with sophisticated behavioural testing and with longitudinal study design aimed at validation across models. 


\section{Acknowledgements}

This work is funded by the Monument Trust Discovery Award from Parkinson's UK (J-1403) to R.W.$\mathrm{M}$. and an EU Joint Programme - Neurodegenerative Disease research program. We thank Nora Bengoa-Vergniory and Brent J. Ryan for critical reading of the manuscript.

1. Pringsheim, T., et al., The prevalence of Parkinson's disease: a systematic review and metaanalysis. Mov Disord, 2014. 29(13): p. 1583-90.

2. Poewe, W., et al., Parkinson disease. Nat Rev Dis Primers, 2017. 3: p. 17013.

3. Schapira, A.H.V., K.R. Chaudhuri, and P. Jenner, Non-motor features of Parkinson disease. Nat Rev Neurosci, 2017. 18(7): p. 435-450.

4. Braak, H., et al., Staging of brain pathology related to sporadic Parkinson's disease. Neurobiol Aging, 2003. 24(2): p. 197-211.

5. Spillantini, M.G., et al., Alpha-synuclein in Lewy bodies. Nature, 1997. 388(6645): p. 839-40.

6. Shahmoradian, S.H., et al., Lewy pathology in Parkinson's disease consists of a crowded organellar membranous medley. bioRxiv, 2017.

7. Trinh, J. and M. Farrer, Advances in the genetics of Parkinson disease. Nat Rev Neurol, 2013. 9(8): p. 445-54.

8. Langston, J.W., et al., Chronic Parkinsonism in humans due to a product of meperidineanalog synthesis. Science, 1983. 219(4587): p. 979-80.

9. Schildknecht, S., et al., Tipping Points and Endogenous Determinants of Nigrostriatal Degeneration by MPTP. Trends Pharmacol Sci, 2017. 38(6): p. 541-555.

10. Meredith, G.E. and D.J. Rademacher, MPTP mouse models of Parkinson's disease: an update. J Parkinsons Dis, 2011. 1(1): p. 19-33.

11. Laloux, C., et al., MPTP-treated mice: long-lasting loss of nigral TH-ir neurons but not paradoxical sleep alterations. Exp Brain Res, 2008. 186(4): p. 635-42.

12. Laloux, C., et al., Effect of dopaminergic substances on sleep/wakefulness in saline-and MPTP-treated mice. J Sleep Res, 2008. 17(1): p. 101-10.

13. Anderson, G., et al., Loss of enteric dopaminergic neurons and associated changes in colon motility in an MPTP mouse model of Parkinson's disease. Exp Neurol, 2007. 207(1): p. 4-12.

14. Tanila, H., M. Bjorklund, and P. Riekkinen, Jr., Cognitive changes in mice following moderate MPTP exposure. Brain Res Bull, 1998. 45(6): p. 577-82.

15. Vuckovic, M.G., et al., Memory, mood, dopamine, and serotonin in the 1-methyl-4-phenyl1,2,3,6-tetrahydropyridine-lesioned mouse model of basal ganglia injury. Neurobiol Dis, 2008. 32(2): p. 319-27.

16. Gibrat, C., et al., Differences between subacute and chronic MPTP mice models: investigation of dopaminergic neuronal degeneration and alpha-synuclein inclusions. J Neurochem, 2009. 109(5): p. 1469-82.

17. Korecka, J.A., et al., Modeling early Parkinson's disease pathology with chronic low dose MPTP treatment. Restor Neurol Neurosci, 2013. 31(2): p. 155-67.

18. Fornai, F., et al., Parkinson-like syndrome induced by continuous MPTP infusion: convergent roles of the ubiquitin-proteasome system and alpha-synuclein. Proc Natl Acad Sci U S A, 2005. 102(9): p. 3413-8.

19. Meredith, G.E., et al., Modeling PD pathogenesis in mice: advantages of a chronic MPTP protocol. Parkinsonism Relat Disord, 2008. 14 Suppl 2: p. S112-5.

20. Munoz-Manchado, A.B., et al., Chronic and progressive Parkinson's disease MPTP model in adult and aged mice. J Neurochem, 2016. 136(2): p. 373-87.

21. Alvarez-Fischer, D., et al., Modelling Parkinson-like neurodegeneration via osmotic minipump delivery of MPTP and probenecid. J Neurochem, 2008. 107(3): p. 701-11. 
22. Shimoji, M., et al., Absence of inclusion body formation in the MPTP mouse model of Parkinson's disease. Brain Res Mol Brain Res, 2005. 134(1): p. 103-8.

23. Goldberg, N.R., et al., Dopaminergic and behavioral correlates of progressive lesioning of the nigrostriatal pathway with 1-methyl-4-phenyl-1,2,3,6-tetrahydropyridine. Neuroscience, 2011. 180: p. 256-71.

24. Petroske, E., et al., Mouse model of Parkinsonism: a comparison between subacute MPTP and chronic MPTP/probenecid treatment. Neuroscience, 2001. 106(3): p. 589-601.

25. Schintu, N., et al., Progressive dopaminergic degeneration in the chronic MPTPp mouse model of Parkinson's disease. Neurotox Res, 2009. 16(2): p. 127-39.

26. Tieu, K., A guide to neurotoxic animal models of Parkinson's disease. Cold Spring Harb Perspect Med, 2011. 1(1): p. a009316.

27. Duty, S. and P. Jenner, Animal models of Parkinson's disease: a source of novel treatments and clues to the cause of the disease. Br J Pharmacol, 2011. 164(4): p. 1357-91.

28. Heuer, A. and S.B. Dunnett, Unilateral 6-OHDA lesions induce lateralised deficits in a 'skinner box' operant choice reaction time task in rats. J Parkinsons Dis, 2012. 2(4): p. 309-20.

29. Lelos, M.J., et al., Amelioration of non-motor dysfunctions after transplantation of human dopamine neurons in a model of Parkinson's disease. Exp Neurol, 2016. 278: p. 54-61.

30. Campos, F.L., et al., Rodent models of Parkinson's disease: beyond the motor symptomatology. Front Behav Neurosci, 2013. 7: p. 175.

31. Cenci, M.A., C.S. Lee, and A. Bjorklund, L-DOPA-induced dyskinesia in the rat is associated with striatal overexpression of prodynorphin- and glutamic acid decarboxylase mRNA. Eur J Neurosci, 1998. 10(8): p. 2694-706.

32. Heikkila, R.E., et al., Dopaminergic toxicity of rotenone and the 1-methyl-4-phenylpyridinium ion after their stereotaxic administration to rats: implication for the mechanism of 1-methyl4-phenyl-1,2,3,6-tetrahydropyridine toxicity. Neurosci Lett, 1985. 62(3): p. 389-94.

33. Betarbet, R., et al., Chronic systemic pesticide exposure reproduces features of Parkinson's disease. Nat Neurosci, 2000. 3(12): p. 1301-6.

34. Johnson, M.E. and L. Bobrovskaya, An update on the rotenone models of Parkinson's disease: their ability to reproduce the features of clinical disease and model gene-environment interactions. Neurotoxicology, 2015. 46: p. 101-16.

35. Lapointe, N., et al., Rotenone induces non-specific central nervous system and systemic toxicity. FASEB J, 2004. 18(6): p. 717-9.

36. Cannon, J.R., et al., A highly reproducible rotenone model of Parkinson's disease. Neurobiol Dis, 2009. 34(2): p. 279-90.

37. Pan-Montojo, F., et al., Progression of Parkinson's disease pathology is reproduced by intragastric administration of rotenone in mice. PLoS One, 2010. 5(1): p. e8762.

38. Gerhard, A., et al., In vivo imaging of microglial activation with [11C](R)-PK11195 PET in idiopathic Parkinson's disease. Neurobiol Dis, 2006. 21(2): p. 404-12.

39. Whitton, P.S., Inflammation as a causative factor in the aetiology of Parkinson's disease. $\mathrm{Br} \mathrm{J}$ Pharmacol, 2007. 150(8): p. 963-76.

40. Tansey, M.G. and M.S. Goldberg, Neuroinflammation in Parkinson's disease: its role in neuronal death and implications for therapeutic intervention. Neurobiol Dis, 2010. 37(3): p. 510-8.

41. Kurkowska-Jastrzebska, I., et al., Decreased inflammation and augmented expression of trophic factors correlate with MOG-induced neuroprotection of the injured nigrostriatal system in the murine MPTP model of Parkinson's disease. Int Immunopharmacol, 2009. 9(6): p. 781-91.

42. Wu, D.C., et al., Blockade of microglial activation is neuroprotective in the 1-methyl-4-phenyl1,2,3,6-tetrahydropyridine mouse model of Parkinson disease. J Neurosci, 2002. 22(5): p. 1763-71. 
43. Sherer, T.B., et al., Selective microglial activation in the rat rotenone model of Parkinson's disease. Neurosci Lett, 2003. 341(2): p. 87-90.

44. Cebrián, C., J.D. Loike, and D. Sulzer, Neuroinflammation in Parkinson's Disease Animal Models: A Cell Stress Response or a Step in Neurodegeneration?, in Behavioral Neurobiology of Huntington's Disease and Parkinson's Disease, H.H.P. Nguyen and M.A. Cenci, Editors. 2015, Springer Berlin Heidelberg: Berlin, Heidelberg. p. 237-270.

45. Tufekci, K.U., S. Genc, and K. Genc, The endotoxin-induced neuroinflammation model of Parkinson's disease. Parkinsons Dis, 2011. 2011: p. 487450.

46. Iravani, M.M., et al., The acute and the long-term effects of nigral lipopolysaccharide administration on dopaminergic dysfunction and glial cell activation. Eur J Neurosci, 2005. 22(2): p. 317-30.

47. Zhang, J., et al., Intrapallidal lipopolysaccharide injection increases iron and ferritin levels in glia of the rat substantia nigra and induces locomotor deficits. Neuroscience, 2005. 135(3): p. 829-38.

48. Zeiss, C.J., H.G. Allore, and A.P. Beck, Established patterns of animal study design undermine translation of disease-modifying therapies for Parkinson's disease. PLoS One, 2017. 12(2): p. e0171790.

49. Marras, C., et al., Nomenclature of genetic movement disorders: Recommendations of the international Parkinson and movement disorder society task force. Mov Disord, 2016. 31(4): p. 436-57.

50. Trempe, J.F. and E.A. Fon, Structure and Function of Parkin, PINK1, and DJ-1, the Three Musketeers of Neuroprotection. Front Neurol, 2013. 4: p. 38.

51. Gispert, S., et al., Parkinson phenotype in aged PINK1-deficient mice is accompanied by progressive mitochondrial dysfunction in absence of neurodegeneration. PLoS One, 2009. 4(6): p. e5777.

52. Goldberg, M.S., et al., Parkin-deficient mice exhibit nigrostriatal deficits but not loss of dopaminergic neurons. J Biol Chem, 2003. 278(44): p. 43628-35.

53. Chandran, J.S., et al., Progressive behavioral deficits in DJ-1-deficient mice are associated with normal nigrostriatal function. Neurobiol Dis, 2008. 29(3): p. 505-14.

54. Itier, J.M., et al., Parkin gene inactivation alters behaviour and dopamine neurotransmission in the mouse. Hum Mol Genet, 2003. 12(18): p. 2277-91.

55. Kitada, T., et al., Absence of nigral degeneration in aged parkin/DJ-1/PINK1 triple knockout mice. J Neurochem, 2009. 111(3): p. 696-702.

56. Dave, K.D., et al., Phenotypic characterization of recessive gene knockout rat models of Parkinson's disease. Neurobiol Dis, 2014. 70: p. 190-203.

57. Cookson, M.R., LRRK2 Pathways Leading to Neurodegeneration. Curr Neurol Neurosci Rep, 2015. 15(7): p. 42.

58. Wallings, R., C. Manzoni, and R. Bandopadhyay, Cellular processes associated with LRRK2 function and dysfunction. FEBS J, 2015. 282(15): p. 2806-26.

59. Yue, M., et al., Progressive dopaminergic alterations and mitochondrial abnormalities in LRRK2 G2019S knock-in mice. Neurobiol Dis, 2015. 78: p. 172-95.

60. Tong, Y., et al., R1441C mutation in LRRK2 impairs dopaminergic neurotransmission in mice. Proc Natl Acad Sci U S A, 2009. 106(34): p. 14622-7.

61. Sloan, M., et al., LRRK2 BAC transgenic rats develop progressive, L-DOPA-responsive motor impairment, and deficits in dopamine circuit function. Hum Mol Genet, 2016. 25(5): p. 95163.

62. Melrose, H.L., et al., Impaired dopaminergic neurotransmission and microtubule-associated protein tau alterations in human LRRK2 transgenic mice. Neurobiol Dis, 2010. 40(3): p. 50317. 
63. $\mathrm{Li}, \mathrm{X}$., et al., Enhanced striatal dopamine transmission and motor performance with LRRK2 overexpression in mice is eliminated by familial Parkinson's disease mutation G2019S. J Neurosci, 2010. 30(5): p. 1788-97.

64. Li, Y., et al., Mutant LRRK2(R1441G) BAC transgenic mice recapitulate cardinal features of Parkinson's disease. Nat Neurosci, 2009. 12(7): p. 826-8.

65. Beccano-Kelly, D.A., et al., LRRK2 overexpression alters glutamatergic presynaptic plasticity, striatal dopamine tone, postsynaptic signal transduction, motor activity and memory. Hum Mol Genet, 2015. 24(5): p. 1336-49.

66. Tong, Y., et al., Loss of leucine-rich repeat kinase 2 causes impairment of protein degradation pathways, accumulation of alpha-synuclein, and apoptotic cell death in aged mice. Proc Natl Acad Sci U S A, 2010. 107(21): p. 9879-84.

67. Hinkle, K.M., et al., LRRK2 knockout mice have an intact dopaminergic system but display alterations in exploratory and motor co-ordination behaviors. Mol Neurodegener, 2012. 7: p. 25.

68. Bonifacino, J.S. and J.H. Hurley, Retromer. Curr Opin Cell Biol, 2008. 20(4): p. 427-36.

69. Wen, L., et al., VPS35 haploinsufficiency increases Alzheimer's disease neuropathology. J Cell Biol, 2011. 195(5): p. 765-79.

70. Tang, F.L., et al., VPS35 Deficiency or Mutation Causes Dopaminergic Neuronal Loss by Impairing Mitochondrial Fusion and Function. Cell Rep, 2015. 12(10): p. 1631-43.

71. Tang, F.L., et al., VPS35 in Dopamine Neurons Is Required for Endosome-to-Golgi Retrieval of Lamp2a, a Receptor of Chaperone-Mediated Autophagy That Is Critical for alpha-Synuclein Degradation and Prevention of Pathogenesis of Parkinson's Disease. J Neurosci, 2015. 35(29): p. 10613-28.

72. Sidransky, E. and G. Lopez, The link between the GBA gene and parkinsonism. Lancet Neurol, 2012. 11(11): p. 986-98.

73. Fernandes, H.J., et al., ER Stress and Autophagic Perturbations Lead to Elevated Extracellular alpha-Synuclein in GBA-N370S Parkinson's iPSC-Derived Dopamine Neurons. Stem Cell Reports, 2016. 6(3): p. 342-56.

74. Richter, F., et al., A GCase chaperone improves motor function in a mouse model of synucleinopathy. Neurotherapeutics, 2014. 11(4): p. 840-56.

75. Rocha, E.M., et al., Glucocerebrosidase gene therapy prevents alpha-synucleinopathy of midbrain dopamine neurons. Neurobiol Dis, 2015. 82: p. 495-503.

76. Fishbein, I., et al., Augmentation of phenotype in a transgenic Parkinson mouse heterozygous for a Gaucher mutation. Brain, 2014. 137(Pt 12): p. 3235-47.

77. Kett, L.R., et al., alpha-Synuclein-independent histopathological and motor deficits in mice lacking the endolysosomal Parkinsonism protein Atp13a2. J Neurosci, 2015. 35(14): p. 572442.

78. Schultheis, P.J., et al., Atp13a2-deficient mice exhibit neuronal ceroid lipofuscinosis, limited alpha-synuclein accumulation and age-dependent sensorimotor deficits. Hum Mol Genet, 2013. 22(10): p. 2067-82.

79. Vingill, S., et al., LOsS of FBXO7 (PARK15) results in reduced proteasome activity and models a parkinsonism-like phenotype in mice. EMBO J, 2016. 35(18): p. 2008-25.

80. Ekstrand, M.I., et al., Progressive parkinsonism in mice with respiratory-chain-deficient dopamine neurons. Proc Natl Acad Sci U S A, 2007. 104(4): p. 1325-30.

81. Ekstrand, M.I. and D. Galter, The MitoPark Mouse - an animal model of Parkinson's disease with impaired respiratory chain function in dopamine neurons. Parkinsonism Relat Disord, 2009. 15 Suppl 3: p. S185-8.

82. Li, X., et al., Cognitive dysfunction precedes the onset of motor symptoms in the MitoPark mouse model of Parkinson's disease. PLoS One, 2013. 8(8): p. e71341. 
83. Villar-Pique, A., T. Lopes da Fonseca, and T.F. Outeiro, Structure, function and toxicity of alpha-synuclein: the Bermuda triangle in synucleinopathies. J Neurochem, 2016. 139 Suppl 1: p. 240-255.

84. Abeliovich, A., et al., Mice lacking alpha-synuclein display functional deficits in the nigrostriatal dopamine system. Neuron, 2000. 25(1): p. 239-52.

85. Cabin, D.E., et al., Synaptic vesicle depletion correlates with attenuated synaptic responses to prolonged repetitive stimulation in mice lacking alpha-synuclein. J Neurosci, 2002. 22(20): p. 8797-807.

86. Anwar, S., et al., Functional alterations to the nigrostriatal system in mice lacking all three members of the synuclein family. J Neurosci, 2011. 31(20): p. 7264-74.

87. Connor-Robson, N., et al., Combinational losses of synucleins reveal their differential requirements for compensating age-dependent alterations in motor behavior and dopamine metabolism. Neurobiol Aging, 2016. 46: p. 107-12.

88. Masliah, E., et al., Dopaminergic loss and inclusion body formation in alpha-synuclein mice: implications for neurodegenerative disorders. Science, 2000. 287(5456): p. 1265-9.

89. Giasson, B.I., et al., Neuronal alpha-synucleinopathy with severe movement disorder in mice expressing A53T human alpha-synuclein. Neuron, 2002. 34(4): p. 521-33.

90. Lee, M.K., et al., Human alpha-synuclein-harboring familial Parkinson's disease-linked Ala-53 --> Thr mutation causes neurodegenerative disease with alpha-synuclein aggregation in transgenic mice. Proc Natl Acad Sci U S A, 2002. 99(13): p. 8968-73.

91. Chesselet, M.F., et al., A progressive mouse model of Parkinson's disease: the Thy1-aSyn ("Line 61") mice. Neurotherapeutics, 2012. 9(2): p. 297-314.

92. Watson, M.B., et al., Regionally-specific microglial activation in young mice over-expressing human wildtype alpha-synuclein. Exp Neurol, 2012. 237(2): p. 318-34.

93. Matsuoka, Y., et al., Lack of nigral pathology in transgenic mice expressing human alphasynuclein driven by the tyrosine hydroxylase promoter. Neurobiol Dis, 2001. 8(3): p. 535-9.

94. Wakamatsu, M., et al., Accumulation of phosphorylated alpha-synuclein in dopaminergic neurons of transgenic mice that express human alpha-synuclein. J Neurosci Res, 2007. 85(8): p. $1819-25$.

95. Gomez-Isla, T., et al., Motor dysfunction and gliosis with preserved dopaminergic markers in human alpha-synuclein A3OP transgenic mice. Neurobiol Aging, 2003. 24(2): p. 245-58.

96. Tofaris, G.K., et al., Pathological changes in dopaminergic nerve cells of the substantia nigra and olfactory bulb in mice transgenic for truncated human alpha-synuclein(1-120): implications for Lewy body disorders. J Neurosci, 2006. 26(15): p. 3942-50.

97. Wakamatsu, M., et al., Selective loss of nigral dopamine neurons induced by overexpression of truncated human alpha-synuclein in mice. Neurobiol Aging, 2008. 29(4): p. 574-85.

98. Emmer, K.L., et al., E46K human alpha-synuclein transgenic mice develop Lewy-like and tau pathology associated with age-dependent, detrimental motor impairment. J Biol Chem, 2011. 286(40): p. 35104-18.

99. Visanji, N.P., et al., $\alpha$-Synuclein-Based Animal Models of Parkinson's Disease: Challenges and Opportunities in a New Era. Trends in Neurosciences, 2016. 39(11): p. 750-762.

100. Janezic, S., et al., Deficits in dopaminergic transmission precede neuron loss and dysfunction in a new Parkinson model. Proc Natl Acad Sci U S A, 2013. 110(42): p. E4016-25.

101. Cannon, J.R., et al., Expression of human E46K-mutated alpha-synuclein in BAC-transgenic rats replicates early-stage Parkinson's disease features and enhances vulnerability to mitochondrial impairment. Exp Neurol, 2013. 240: p. 44-56.

102. Taylor, T.N., et al., Region-specific deficits in dopamine, but not norepinephrine, signaling in a novel A30P alpha-synuclein BAC transgenic mouse. Neurobiol Dis, 2014. 62: p. 193-207.

103. Kuo, Y.M., et al., Extensive enteric nervous system abnormalities in mice transgenic for artificial chromosomes containing Parkinson disease-associated alpha-synuclein gene 
mutations precede central nervous system changes. Hum Mol Genet, 2010. 19(9): p. 163350.

104. Van der Perren, A., C. Van den Haute, and V. Baekelandt, Viral vector-based models of Parkinson's disease. Curr Top Behav Neurosci, 2015. 22: p. 271-301.

105. Gorbatyuk, O.S., et al., The phosphorylation state of Ser-129 in human alpha-synuclein determines neurodegeneration in a rat model of Parkinson disease. Proc Natl Acad Sci U S A, 2008. 105(2): p. 763-8.

106. Azeredo da Silveira, S., et al., Phosphorylation does not prompt, nor prevent, the formation of alpha-synuclein toxic species in a rat model of Parkinson's disease. Hum Mol Genet, 2009. 18(5): p. 872-87.

107. Daniel, G., et al., alpha-Synuclein-induced dopaminergic neurodegeneration in a rat model of Parkinson's disease occurs independent of ATP13A2 (PARK9). Neurobiol Dis, 2015. 73: p. 22943.

108. Decressac, M., et al., Progressive neurodegenerative and behavioural changes induced by AAV-mediated overexpression of alpha-synuclein in midbrain dopamine neurons. Neurobiol Dis, 2012. 45(3): p. 939-53.

109. Yamada, M., et al., Overexpression of alpha-synuclein in rat substantia nigra results in loss of dopaminergic neurons, phosphorylation of alpha-synuclein and activation of caspase-9: resemblance to pathogenetic changes in Parkinson's disease. J Neurochem, 2004. 91(2): p. 451-61.

110. Ulusoy, A., et al., Co-expression of C-terminal truncated alpha-synuclein enhances full-length alpha-synuclein-induced pathology. Eur J Neurosci, 2010. 32(3): p. 409-22.

111. Volpicelli-Daley, L.A., et al., How can rAAV-alpha-synuclein and the fibril alpha-synuclein models advance our understanding of Parkinson's disease? J Neurochem, 2016. 139 Suppl 1: p. 131-155.

112. Gombash, S.E., et al., Morphological and behavioral impact of AAV2/5-mediated overexpression of human wildtype alpha-synuclein in the rat nigrostriatal system. PLoS One, 2013. 8(11): p. e81426.

113. Winner, B., et al., In vivo demonstration that alpha-synuclein oligomers are toxic. Proc Natl Acad Sci U S A, 2011. 108(10): p. 4194-9.

114. Eslamboli, A., et al., Long-term consequences of human alpha-synuclein overexpression in the primate ventral midbrain. Brain, 2007. 130(Pt 3): p. 799-815.

115. Oliveras-Salva, M., et al., rAAV2/7 vector-mediated overexpression of alpha-synuclein in mouse substantia nigra induces protein aggregation and progressive dose-dependent neurodegeneration. Mol Neurodegener, 2013. 8: p. 44.

116. Van der Perren, A., et al., Longitudinal follow-up and characterization of a robust rat model for Parkinson's disease based on overexpression of alpha-synuclein with adeno-associated viral vectors. Neurobiol Aging, 2015. 36(3): p. 1543-58.

117. Bourdenx, M., et al., Lack of additive role of ageing in nigrostriatal neurodegeneration triggered by alpha-synuclein overexpression. Acta Neuropathol Commun, 2015. 3: p. 46.

118. Koprich, J.B., et al., Progressive neurodegeneration or endogenous compensation in an animal model of Parkinson's disease produced by decreasing doses of alpha-synuclein. PLoS One, 2011. 6(3): p. e17698.

119. Gaugler, M.N., et al., Nigrostriatal overabundance of alpha-synuclein leads to decreased vesicle density and deficits in dopamine release that correlate with reduced motor activity. Acta Neuropathol, 2012. 123(5): p. 653-69.

120. Decressac, M., B. Mattsson, and A. Bjorklund, Comparison of the behavioural and histological characteristics of the 6-OHDA and alpha-synuclein rat models of Parkinson's disease. Exp Neurol, 2012. 235(1): p. 306-15. 
121. Tozzi, A., et al., Alpha-Synuclein Produces Early Behavioral Alterations via Striatal Cholinergic Synaptic Dysfunction by Interacting With GluN2D N-Methyl-D-Aspartate Receptor Subunit. Biol Psychiatry, 2016. 79(5): p. 402-14.

122. St Martin, J.L., et al., Dopaminergic neuron loss and up-regulation of chaperone protein mRNA induced by targeted over-expression of alpha-synuclein in mouse substantia nigra. J Neurochem, 2007. 100(6): p. 1449-57.

123. Ulusoy, A., et al., Dysregulated dopamine storage increases the vulnerability to alphasynuclein in nigral neurons. Neurobiol Dis, 2012. 47(3): p. 367-77.

124. Song, L.K., et al., Targeted Overexpression of alpha-Synuclein by rAAV2/1 Vectors Induces Progressive Nigrostriatal Degeneration and Increases Vulnerability to MPTP in Mouse. PLoS One, 2015. 10(6): p. e0131281.

125. Kordower, J.H., et al., Lewy body-like pathology in long-term embryonic nigral transplants in Parkinson's disease. Nat Med, 2008. 14(5): p. 504-6.

126. Recasens, A. and B. Dehay, Alpha-synuclein spreading in Parkinson's disease. Front Neuroanat, 2014. 8: p. 159.

127. Luk, K.C., et al., Intracerebral inoculation of pathological alpha-synuclein initiates a rapidly progressive neurodegenerative alpha-synucleinopathy in mice. J Exp Med, 2012. 209(5): p. 975-86.

128. Mougenot, A.L., et al., Prion-like acceleration of a synucleinopathy in a transgenic mouse model. Neurobiol Aging, 2012. 33(9): p. 2225-8.

129. Watts, J.C., et al., Transmission of multiple system atrophy prions to transgenic mice. Proc Natl Acad Sci U S A, 2013. 110(48): p. 19555-60.

130. Recasens, A., et al., Lewy body extracts from Parkinson disease brains trigger alpha-synuclein pathology and neurodegeneration in mice and monkeys. Ann Neurol, 2014. 75(3): p. 351-62.

131. Masuda-Suzukake, M., et al., Prion-like spreading of pathological alpha-synuclein in brain. Brain, 2013. 136(Pt 4): p. 1128-38.

132. Luk, K.C., et al., Pathological alpha-synuclein transmission initiates Parkinson-like neurodegeneration in nontransgenic mice. Science, 2012. 338(6109): p. 949-53.

133. Sacino, A.N., et al., Brain injection of alpha-synuclein induces multiple proteinopathies, gliosis, and a neuronal injury marker. J Neurosci, 2014. 34(37): p. 12368-78.

134. Masuda-Suzukake, M., et al., Pathological alpha-synuclein propagates through neural networks. Acta Neuropathol Commun, 2014. 2: p. 88.

135. Hansen, C., et al., alpha-Synuclein propagates from mouse brain to grafted dopaminergic neurons and seeds aggregation in cultured human cells. J Clin Invest, 2011. 121(2): p. 715-25.

136. Sacino, A.N., et al., Induction of CNS alpha-synuclein pathology by fibrillar and nonamyloidogenic recombinant alpha-synuclein. Acta Neuropathol Commun, 2013. 1: p. 38.

137. Osterberg, V.R., et al., Progressive aggregation of alpha-synuclein and selective degeneration of lewy inclusion-bearing neurons in a mouse model of parkinsonism. Cell Rep, 2015. 10(8): p. 1252-60.

138. Luk, K.C., et al., Molecular and Biological Compatibility with Host Alpha-Synuclein Influences Fibril Pathogenicity. Cell Rep, 2016. 16(12): p. 3373-87.

139. Abdelmotilib, $\mathrm{H}_{\text {., }}$ et al., $\alpha$-Synuclein fibril-induced inclusion spread in rats and mice correlates with dopaminergic Neurodegeneration. Neurobiology of Disease, 2017. 105: p. 84-98.

140. Sorrentino, Z.A., et al., Intrastriatal injection of alpha-synuclein can lead to widespread synucleinopathy independent of neuroanatomic connectivity. Mol Neurodegener, 2017. 12(1): p. 40.

141. Ayers, J.I., et al., Robust Central Nervous System Pathology in Transgenic Mice following Peripheral Injection of alpha-Synuclein Fibrils. J Virol, 2017. 91(2).

142. Mason, D.M., et al., Transmission of alpha-synucleinopathy from olfactory structures deep into the temporal lobe. Mol Neurodegener, 2016. 11(1): p. 49. 
143. Sacino, A.N., et al., Amyloidogenic alpha-synuclein seeds do not invariably induce rapid, widespread pathology in mice. Acta Neuropathol, 2014. 127(5): p. 645-65.

144. Peelaerts, W., et al., alpha-Synuclein strains cause distinct synucleinopathies after local and systemic administration. Nature, 2015. 522(7556): p. 340-4.

145. Sacino, A.N., et al., Intramuscular injection of alpha-synuclein induces CNS alpha-synuclein pathology and a rapid-onset motor phenotype in transgenic mice. Proc Natl Acad Sci U S A, 2014. 111(29): p. 10732-7.

146. Holmqvist, S., et al., Direct evidence of Parkinson pathology spread from the gastrointestinal tract to the brain in rats. Acta Neuropathol, 2014. 128(6): p. 805-20.

147. Fortuna, J.T.S., et al., Brain infusion of alpha-synuclein oligomers induces motor and nonmotor Parkinson's disease-like symptoms in mice. Behav Brain Res, 2017.

148. Rey, N.L., et al., Transfer of human alpha-synuclein from the olfactory bulb to interconnected brain regions in mice. Acta Neuropathol, 2013. 126(4): p. 555-73.

149. Rey, N.L., et al., Widespread transneuronal propagation of alpha-synucleinopathy triggered in olfactory bulb mimics prodromal Parkinson's disease. J Exp Med, 2016. 213(9): p. 1759-78.

150. Paumier, K.L., et al., Intrastriatal injection of pre-formed mouse alpha-synuclein fibrils into rats triggers alpha-synuclein pathology and bilateral nigrostriatal degeneration. Neurobiol Dis, 2015. 82: p. 185-99.

151. Abdelmotilib, H., et al., alpha-Synuclein fibril-induced inclusion spread in rats and mice correlates with dopaminergic Neurodegeneration. Neurobiol Dis, 2017. 105: p. 84-98.

152. Hilker, R., et al., Nonlinear progression of Parkinson disease as determined by serial positron emission tomographic imaging of striatal fluorodopa F 18 activity. Arch Neurol, 2005. 62(3): p. 378-82.

153. Morrish, P.K., et al., Measuring the rate of progression and estimating the preclinical period of Parkinson's disease with [18F]dopa PET. J Neurol Neurosurg Psychiatry, 1998. 64(3): p. 314-9.

154. Fearnley, J.M. and A.J. Lees, Ageing and Parkinson's disease: substantia nigra regional selectivity. Brain, 1991. 114 ( Pt 5): p. 2283-301.

155. Schirinzi, T., et al., Early synaptic dysfunction in Parkinson's disease: Insights from animal models. Mov Disord, 2016. 31(6): p. 802-13.

156. Lam, H.A., et al., Elevated tonic extracellular dopamine concentration and altered dopamine modulation of synaptic activity precede dopamine loss in the striatum of mice overexpressing human alpha-synuclein. J Neurosci Res, 2011. 89(7): p. 1091-102.

157. Sossi, V., et al., Dopamine turnover increases in asymptomatic LRRK2 mutations carriers. Mov Disord, 2010. 25(16): p. 2717-23.

158. Ritz, B.R., et al., Dopamine transporter genetic variants and pesticides in Parkinson's disease. Environ Health Perspect, 2009. 117(6): p. 964-9.

159. Caudle, W.M., et al., Reduced vesicular storage of dopamine causes progressive nigrostriatal neurodegeneration. J Neurosci, 2007. 27(30): p. 8138-48.

160. Ellenbroek, B. and J. Youn, Rodent models in neuroscience research: is it a rat race? Dis Model Mech, 2016. 9(10): p. 1079-1087.

161. Nuber, S., et al., A progressive dopaminergic phenotype associated with neurotoxic conversion of alpha-synuclein in BAC-transgenic rats. Brain, 2013. 136(Pt 2): p. 412-32.

162. Rey, N.L., S. George, and P. Brundin, Spreading the word: Precise animal models and validated methods are vital when evaluating prion-like behaviour of alpha-synuclein. Neuropathology and Applied Neurobiology, 2016. 42(1): p. 51-76.

163. Dehay, B., et al., Alpha-synuclein propagation: New insights from animal models. Mov Disord, 2016. 31(2): p. 161-8.

164. Sulzer, D., Multiple hit hypotheses for dopamine neuron loss in Parkinson's disease. Trends Neurosci, 2007. 30(5): p. 244-50. 
165. Kim, R.H., et al., Hypersensitivity of DJ-1-deficient mice to 1-methyl-4-phenyl-1,2,3,6tetrahydropyrindine (MPTP) and oxidative stress. Proc Natl Acad Sci U S A, 2005. 102(14): p. 5215-20.

166. Manning-Bog, A.B., et al., Increased vulnerability of nigrostriatal terminals in DJ-1-deficient mice is mediated by the dopamine transporter. Neurobiol Dis, 2007. 27(2): p. 141-50.

167. Oliveras-Salva, M., et al., Alpha-synuclein-induced neurodegeneration is exacerbated in PINK1 knockout mice. Neurobiol Aging, 2014. 35(11): p. 2625-36.

168. Dauer, W., et al., Resistance of alpha -synuclein null mice to the parkinsonian neurotoxin MPTP. Proc Natl Acad Sci U S A, 2002. 99(22): p. 14524-9.

169. Schluter, O.M., et al., Role of alpha-synuclein in 1-methyl-4-phenyl-1,2,3,6tetrahydropyridine-induced parkinsonism in mice. Neuroscience, 2003. 118(4): p. 985-1002.

170. Robertson, D.C., et al., Developmental loss and resistance to MPTP toxicity of dopaminergic neurones in substantia nigra pars compacta of gamma-synuclein, alpha-synuclein and double alpha/gamma-synuclein null mutant mice. J Neurochem, 2004. 89(5): p. 1126-36.

171. Mulcahy, P., et al., The behavioural and neuropathological impact of intranigral AAV-alphasynuclein is exacerbated by systemic infusion of the Parkinson's disease-associated pesticide, rotenone, in rats. Behav Brain Res, 2013. 243: p. 6-15.

172. Volpicelli-Daley, L.A., et al., G2019s-LRRK2 expression augments $\alpha$-synuclein sequestration into inclusions in neurons. Journal of Neuroscience, 2016. 36(28): p. 7415-7427.

173. Deleidi, M., et al., The Toll-like receptor-3 agonist polyinosinic:polycytidylic acid triggers nigrostriatal dopaminergic degeneration. J Neurosci, 2010. 30(48): p. 16091-101.

174. Bobyn, J., et al., Viral-toxin interactions and Parkinson's disease: poly I:C priming enhanced the neurodegenerative effects of paraquat. J Neuroinflammation, 2012. 9: p. 86.

175. Frank-Cannon, T.C., et al., Parkin deficiency increases vulnerability to inflammation-related nigral degeneration. J Neurosci, 2008. 28(43): p. 10825-34.

176. Gao, H.M., et al., Neuroinflammation and alpha-synuclein dysfunction potentiate each other, driving chronic progression of neurodegeneration in a mouse model of Parkinson's disease. Environ Health Perspect, 2011. 119(6): p. 807-14.

177. Gao, H.M., et al., Neuroinflammation and oxidation/nitration of alpha-synuclein linked to dopaminergic neurodegeneration. J Neurosci, 2008. 28(30): p. 7687-98.

178. Moehle, M.S., et al., LRRK2 inhibition attenuates microglial inflammatory responses. J Neurosci, 2012. 32(5): p. 1602-11.

179. Wahlsten, D., et al., Different data from different labs: lessons from studies of geneenvironment interaction. J Neurobiol, 2003. 54(1): p. 283-311.

180. Crawley, J.N., Behavioral phenotyping strategies for mutant mice. Neuron, 2008. 57(6): p. 809-18.

181. Kilkenny, C., et al., Improving bioscience research reporting: the ARRIVE guidelines for reporting animal research. PLoS Biol, 2010. 8(6): p. e1000412.

182. Richardson, C.A., The power of automated behavioural homecage technologies in characterizing disease progression in laboratory mice: A review. Applied Animal Behaviour Science, 2015. 163: p. 19-27.

183. Bains, R.S., et al., Assessing mouse behaviour throughout the light/dark cycle using automated in-cage analysis tools. J Neurosci Methods, 2017.

184. Geldenhuys, W.J., et al., A novel biomechanical analysis of gait changes in the MPTP mouse model of Parkinson's disease. PeerJ, 2015. 3: p. e1175.

185. Datto, J.P., et al., Use of the CatWalk Gait Device to Assess Differences in Locomotion between Genders in Rats Inherently and following Spinal Cord Injury. Dataset Papers in Science, 2016. 2016: p. 11.

186. Horner, A.E., et al., The touchscreen operant platform for testing learning and memory in rats and mice. Nat Protoc, 2013. 8(10): p. 1961-84. 
187. Paumier, K.L., et al., Behavioral characterization of A53T mice reveals early and late stage deficits related to Parkinson's disease. PLoS One, 2013. 8(8): p. e70274.

188. Schrag, A., M. Jahanshahi, and N. Quinn, What contributes to quality of life in patients with Parkinson's disease? J Neurol Neurosurg Psychiatry, 2000. 69(3): p. 308-12.

189. Taylor, T.N., J.G. Greene, and G.W. Miller, Behavioral phenotyping of mouse models of Parkinson's disease. Behav Brain Res, 2010. 211(1): p. 1-10.

190. Cummings, J.L., T. Morstorf, and K. Zhong, Alzheimer's disease drug-development pipeline: few candidates, frequent failures. Alzheimers Res Ther, 2014. 6(4): p. 37.

191. Ninkina, N., et al., A novel resource for studying function and dysfunction of alpha-synuclein: mouse lines for modulation of endogenous Snca gene expression. Sci Rep, 2015. 5: p. 16615. 